\title{
Boundary Value Problem of an Infinite Array of Loaded Apertures
}

\author{
Luis Alejandro Iturri-Hinojosa ${ }^{1}$, Alexander E. Martynyuk ${ }^{2}$ \\ and Mohamed Badaoui ${ }^{1}$ \\ ${ }^{1}$ National Polytechnic Institute, IPN, Mexico DF, E-mail: aiturrih@gmail.com \\ ${ }^{2}$ Faculty of Engineering, National Autonomous University of Mexico (UNAM), Mexico
}

\begin{abstract}
Keywords: Loaded-aperture cells, phased array antennas, Floquet's theorem and RADANT principle.
\end{abstract}

\begin{abstract}
A mathematical model of the scattering by a periodically arranged apertures in conducting plates is presented. The boundary value problem of an infinite array of loaded apertures is formulated for an arbitrary incident plane wave. The reflection coefficient for some array geometries is obtained and the calculated values are in good agreement with the measurements in a previously published researches. All the rectangular apertures in the array are assumed to be identical and infinitesimally thin. The mathematical model is based on Floquet's theorem that specifies the requirement of periodicity by the electromagnetic fields.
\end{abstract}

\section{Introduction}

A mathematical model for the design of periodic rectangular array based on loaded aperture cells had been developed. The formulation of the tangential electric field induced on the apertures is the basis of the multilayer arrays operation, well-known RADANT principle. Each layer of the RADANT lens consists of small cells arranged at the nodes of the rectangular periodic grid. The small cell is a rectangular capacitive aperture loaded with $\mathrm{p}-\mathrm{i}-\mathrm{n}$ diodes. The equivalent reactance of the layer can be changed by proper commutation of the diodes within the cells. The different reactances of the layer assure the different phase shifts introduced in the plane wave transmitted through the layer [1]. Thus, it is possible to add a linearly-distributed phase shift across the incident plane wave and to redirect it in a desired direction [2].

To predict the effect of the strong mutual coupling between the cells, a full-wave mathematical model had been developed. The normally-incident linearly polarized wave had been considered as an excitation source.

If the biases across all p-i-n diodes are the same for all the cells in the layer, this layer can be analyzed as a periodic structure using the theorem of Floquet [1-3]. The small cell is considered as a unit cell of this periodic structure. However, in this case a constant phase shift is introduced across the transmitted wave and this wave is not redirected. Thus, the different biases applied across the diodes are required for scanning.

The different biases across the diodes destroy the periodicity of the layer. However, the layer can be treated as a periodic structure with a large unit cell that contains $M$ adjacent small cells in the $\mathrm{x}$ direction per $\mathrm{N}$ adjacent small cells in the $\mathrm{y}$-direction when this large unit cell is repeated periodically along the layer. Thus, the layer can be analyzed as infinite periodic structure with the help of Floquet's theorem. Application of the boundary condition for the magnetic intensity vector together with the Ohm's law on the diodes within a large unit cell leads to the system of integral equations with respect to the unknown tangential electric intensity vector. This system of integral equations had been resolved using the method of moments. As a result, the electromagnetic field in the unit cell had been presented as a sum of Floquet modes or plane waves. 


\section{Mathematical Model Un-loaded unit cell}

Consider the un-loaded unit cell of figure 1. The tangential electric field can be expressed as a sum of Floquet modes:

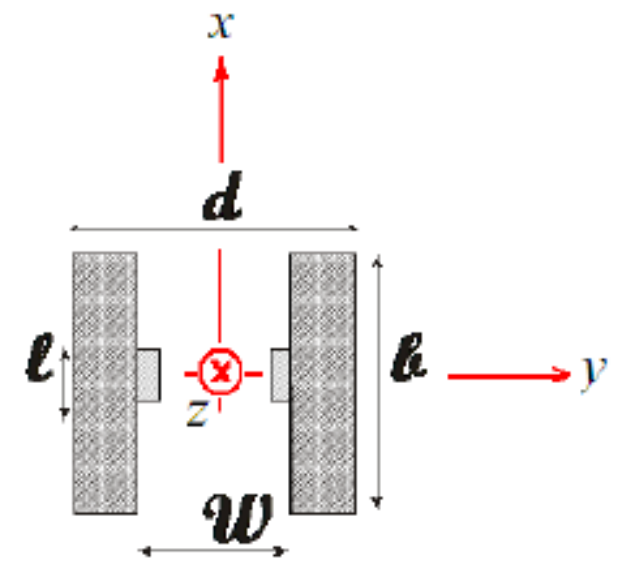

Fig. 1 Un-loaded unit cell

For $\mathrm{z}<0$ :

$$
E_{\tau}(x, y)=\psi_{l 00}(x, y) e^{-j k_{200} z}+\Gamma_{l 00} \psi_{l 00}(x, y) e^{j k_{z i 00} z}+\sum_{2} \sum_{2} \Gamma_{l m n} \psi_{l m n}(x, y) e^{j k_{2 \operatorname{lnm}} z}
$$

and for $\mathrm{z}>0$ :

$$
\vec{E}_{\tau}(x, y)=\sum_{1} T_{l m n} \vec{\Psi}_{l m n}(x, y) e^{-j k_{k_{m m n}} z}
$$

Here the Floquet modes are expressed as:

$\Psi_{m n}^{I E}(x, y)=\sqrt{\frac{1}{b d}}\left(\frac{k_{y n} \vec{x}-k_{x m} \vec{y}}{k_{r m n}}\right) e^{j\left(k_{x w m} x+k_{y m} y\right)} ; \Psi_{m n}^{T M}(x, y)=\sqrt{\frac{1}{b d}}\left(\frac{k_{x m} \vec{x}+k_{y n} \hat{y}}{k_{r m n}}\right) e^{j\left(k_{x m} x+k_{y n} y\right)}$

and $l=1$ or 2 represents the transverse electric and magnetic fields, respectively.

In this case: $1+\Gamma_{1}=T_{1}$ for $\mathrm{n}=1$, and $\Gamma_{n}=T_{n}$ for $\mathrm{n}>1$.

Let consider the boundary condition: $\left(\imath x \vec{H}_{\tau}^{+}\right)-\left(\varepsilon x \vec{H}_{\tau}^{-}\right)=\vec{J}_{s}^{v}=0$.

Equations 1, 2, 3 into 4 (and js $=0, \mathrm{z}=0$ ) we obtain the following expression:

$$
2 Y_{l 00} \psi_{l 00}(x, y)=\sum_{1} \sum_{m} \sum_{n} \Gamma_{l m n}\left(Y_{l m n}+Y_{l m n}^{S}\right) \psi_{l m n}(x, y)
$$

Taking the inverse form of eq. 2 for $\mathrm{m}$ and $\mathrm{n} \geq 2$ and $\mathrm{z}=0$ :

$\Gamma_{l m n}=\int_{S} E_{\tau}(x, y) \cdot \Psi_{l m n}^{*}(x, y) d x d y$

From (6) and (5) we obtain a first order Fredholm equation for the electric tangential field $\vec{E}_{\tau}(x, y)$ in the first unit cell of the large cell:

$$
\iint_{S^{\prime}} \sum_{l} \sum_{m} \sum_{n}\left(Y_{m}+Y_{m}^{d}\right) \vec{\psi}_{m}(x, y) \vec{\psi}_{m}^{*}\left(x^{\prime}, y^{\prime}\right) E_{\tau}(x, y) d x^{\prime} d y^{\prime}=2 Y_{l 00} \vec{\psi}_{l 00}(x, y)
$$

We applied the method of moments to solve the integral equation (7) considering:

$$
E_{\tau}(x, y)=\sum_{1} a_{n}\left[z \times \vec{f}_{p}(x, y)\right]
$$

The following system of linear equations is obtained:

$$
\sum_{l} \sum_{m} \sum_{n}\left(Y_{m}+Y_{m}^{d}\right) \sum_{p} a_{p} C_{l m n}^{p^{*}} C_{l m n}^{q}=2 Y_{l 00} \iint_{S} \vec{f}_{p}(x, y)\left[\psi_{l 00}(x, y) \times \hat{z}\right] d x d y
$$

Where 


$$
C_{l m n}^{p^{*}}=\iint_{S} \vec{f}_{p}(x, y)\left[\vec{\Psi}_{l m n}^{*}(x, y) \times \hat{z}\right] d x^{\prime} d y^{\prime} ; C_{l m n}^{q}=\iint_{S} \vec{f}_{p}(x, y)\left[\vec{\Psi}_{l m n}(x, y) \times \hat{z}\right] d x^{\prime} d y^{\prime}
$$

The basis function $f_{p}$ that we consider for the current distribution induced on the surface is:

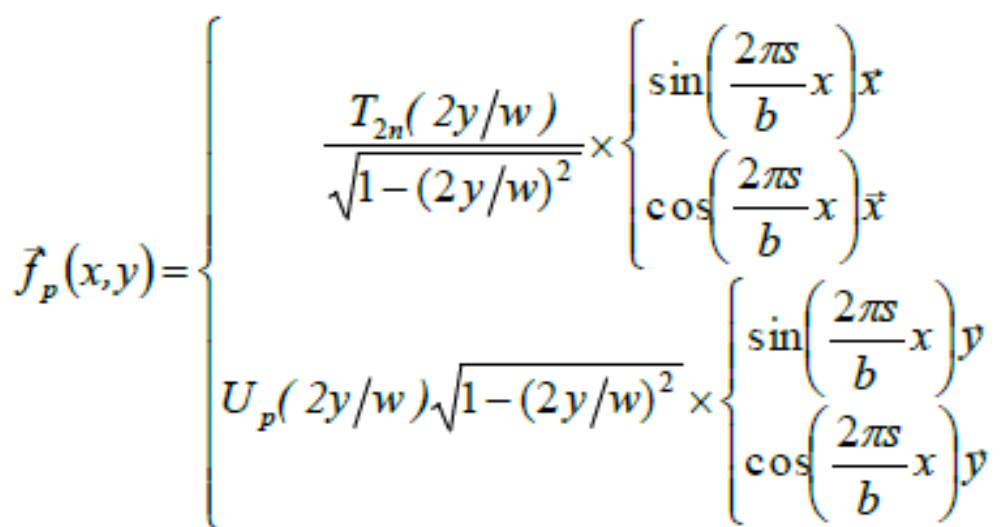

where $p=1,2,3, \ldots, Q$ and $s=1,2,3, \ldots, S$.

Taking the inverse form of (2) and considering equations (8) and (3) we obtain the expression of the reflection coefficient for the perpendicular incident field to the array:

$$
\Gamma_{l 00}=\iint_{S} \sum_{p} a_{p} \vec{f}_{p}(x, y)\left[\vec{\psi}_{l m n}^{*}(x, y) \times \hat{z}\right] d x^{\prime} d y^{\prime}-1
$$

\section{Loaded unit cell}

Considering a load in the aperture of the small cell in figure 2:

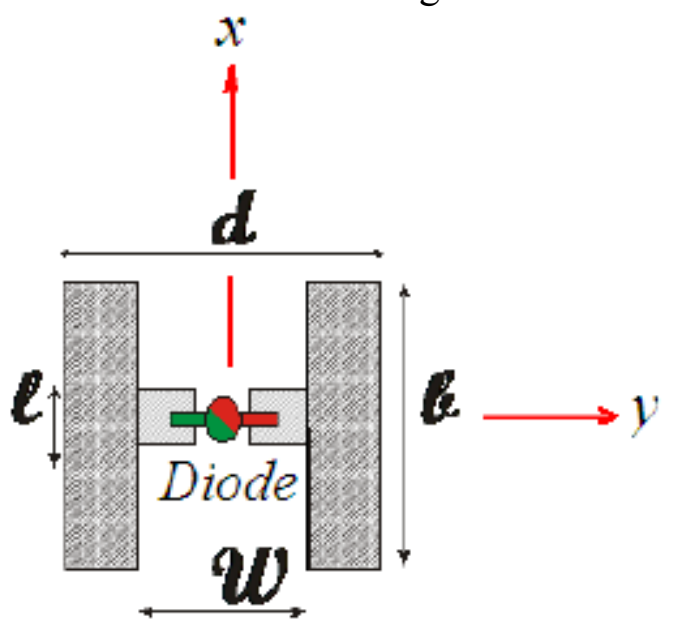

Fig. 2 Loaded aperture small cell

The tangential electric field $\vec{E}_{\tau}(x, y)$ can be expressed by the sum of the un-loaded electric field and the loaded electric field:

$$
\vec{E}_{\tau}(x, y)=\vec{E}_{\tau}^{s d}(x, y)+\vec{E}_{\tau}^{c d}(x, y)
$$

where the first term is the tangential electric field with switches in high impedance state and the second term is the tangential electric field due to induced voltage in PIN diodes.

Considering the boundary condition:

$$
\left(z x \vec{H}_{\tau}^{+}\right)-\left(\approx x \vec{H}_{\tau}^{-}\right)=\vec{j}_{s}^{c d}(\mathrm{x}, \mathrm{y})
$$

Since

$$
\hat{z} x \vec{H}_{\tau}^{+}=-\sum_{l} \sum_{m} \sum_{n} T_{l m n} Y_{l m n}^{s} \vec{\Psi}_{l m n}(x, y) \text { and } \hat{z} x \vec{H}_{\tau}^{-}=\sum_{l} \sum_{m} \sum_{n} \Gamma_{m} Y_{m} \vec{\Psi}_{m}(x, y)
$$


We leads to the following equation:

$\sum_{l} \sum_{m} \sum_{n} \Gamma_{l m n}\left(Y_{l m n}+Y_{l m n}^{S}\right) \vec{\psi}_{l m n}(\mathrm{x}, \mathrm{y})=-J_{s}^{c d}(x, y)$

Where $\Gamma_{l m n}=\int_{s} E_{\tau}^{c d} \cdot \Psi_{l m n}^{*}(x, y) d s$.

Taking into account the reflection coefficient and the Ohms law:

$$
V=\int_{-w / 2}^{w / 2}\left(E_{\tau}^{c d}(x, y)+E_{\tau}^{s d}(x, y)\right) d y=I / Y
$$

We can find the following integral equation:

$$
\iint_{S^{\prime}} \sum \sum \sum\left[\left(Y_{l m n}+Y_{l m n}^{s}\right) \psi_{l m n}(x, y) \psi_{l m n}^{*}(x, y)\right] \vec{E}_{\tau}^{c d}\left(x^{\prime}, y^{\prime}\right) d x^{\prime} d y^{\prime}=-\frac{V Y}{l} y
$$

The reflection coefficient is calculated using equation (10).

For the tangential electric field $\vec{E}_{\tau}^{c d}(x, y)=\sum_{p=1}^{p} a_{p}^{c d}\left[\vec{z} \times \vec{f}_{p}^{c d}(x, y)\right]$ and applying the method of moments we found the linear system equation for the $a_{p}^{c d}$ coefficients:

$$
\begin{gathered}
\sum_{p=1}^{P} a_{p}^{c d}\left[\sum_{l=1}^{2} \sum_{m=-M}^{M} \sum_{n=-N}^{N}\left(Y_{l m n}^{s}+Y_{l m n}\right) \int_{s^{\prime}} \vec{f}_{q}^{c d}\left(x^{\prime}, y^{\prime}\right) \cdot\left[\vec{\Psi}_{l m n}\left(x^{\prime}, y^{\prime}\right) \times \vec{z}\right] d s^{\prime} \int_{s^{\prime}} \vec{f}_{p}^{c d}\left(x^{\prime}, y^{\prime}\right)\right. \\
\left.\cdot\left[\vec{\Psi}_{l m n}^{*}\left(x^{\prime}, y^{\prime}\right) \times \vec{z}\right] d s^{\prime}+\frac{Y}{l} \int_{-w / 2}^{w / 2}\left[\vec{z} \times \vec{f}_{p}^{c d}(x, y)\right] d y \int_{s} \vec{f}_{q x}^{c d}(x, y) d s\right] \\
=-\frac{Y}{l} \sum_{p=1}^{p} a_{p}^{s d} \int_{-w / 2}^{w / 2}\left[\vec{z} \times \vec{f}_{p}^{s d}(x, y)\right] d y \int_{s} \vec{f}_{q x}^{c d}(x, y) d s
\end{gathered}
$$

$\mathrm{q}=1,2,3, \ldots, \mathrm{P}$

where $Y_{l m n}$ are the modal admittances of the Floquet modes in the $z<0$ region. The dielectric substrate is modeled by a transmission line of ${ }^{d_{s}}$ length finished in a load of admittance $\bar{Y}_{l m n}^{s}$ and $\vec{z}$ is the unit vector in $\mathrm{Z}$ direction.

The modal admittance in $z>0$ is represented by:

$$
Y_{l m n}^{s}=\bar{Y}_{l m n}^{s} \frac{Y_{l m n}+j \bar{Y}_{l m n}^{s} \tan \left(k_{m n}^{z s} d_{s}\right)}{\bar{Y}_{l m n}^{s}+j Y_{l m n} \tan \left(k_{m n}^{z s} d_{s}\right)}
$$

The electric and transversal fields are related with the modal admittances by:

$$
Y_{1 m n}=\frac{k_{m n}^{z}}{k} \sqrt{\frac{\varepsilon_{0}}{\mu_{0}}} \quad \bar{Y}_{1 m n}^{s}=\frac{k_{m n}^{z s}}{k^{s}} \sqrt{\frac{\epsilon_{0} \epsilon_{r}}{\mu_{0}}} \quad \quad Y_{2 m n}=\frac{k}{k_{m n}^{z}} \sqrt{\frac{\varepsilon_{0}}{\mu_{0}}} \quad \bar{Y}_{2 m n}^{s}=\frac{k^{s}}{k_{m n}^{z s}} \sqrt{\frac{\epsilon_{0} \epsilon_{r}}{\mu_{0}}}
$$

Where $k_{m n}^{z s}$ is the modal propagation constant in the dielectric substrate at the z-axis represented by $\quad k_{m n}^{z s}=\sqrt{k^{2}-k_{r m n}^{2}}$, where $\quad k_{r m n}^{2}=k_{x m}^{2}+k_{y n}^{2} . \quad k_{x m}=\frac{2 \pi m-\varphi_{x}}{b}, \quad k_{y n}=\frac{2 \pi n-\varphi_{y}}{d}$, $\varphi_{x}=k \sin \theta \cos \phi, \varphi_{y}=k \sin \theta \sin \phi, k=\omega \sqrt{\mu \varepsilon}$ is the wave number and $\varepsilon_{0}, \mu_{0}$ are the electric permittivity and magnetic permeability of free space, respectively.

\section{Acknowledgments}

This material is based upon work supported by the SIP Project 20141285. 


\section{Conclusion}

A mathematical model has been formulated for the analysis of the boundary value problem of an infinite array of loaded apertures. The system integral equations for the tangential electric fields of un-loaded and loaded unit cells were found. The electromagnetic field in the unit cell had been presented as a sum of Floquet modes.

\section{References}

[1] Lau, J.Y. and Hum, S.V., "Reconfigurable Transmit array Design Approaches for Beam forming Applications", IEEE Transactions on Antennas and Propagation, vol. 60, No. 12, Dec. 2012.

[2] C. Chekroun, D. Herric, Y. Michel, R. Pauchard, and P. Vidal, "Radant: New method of electronic scanning," Microw. J., vol. 24, No. 2, pp. 45-53, Feb. 1981.

[3] Benedikt A. Munk, Robert G. Kouyoujian, and Leon Peters, "Reflection Properties of Periodic Surfaces of Loaded Dipoles", IEEE Trans. on Antennas and Propagation, vol. AP-19, No. 5, Sept. 1971. 